Research Paper

\title{
A Prognostic Model Based on Circulating Tumour Cells is Useful for Identifying the Poorest Survival Outcome in Patients with Metastatic Colorectal Cancer
}

\author{
Wen-Chi Chou ${ }^{1,2^{*}}$, Min-Hsien Wu 1 $^{13,4^{*}}$, Pei-Hung Chang ${ }^{5,6^{*}}$, Hung-Chih Hsu1, Gwo-jyh Chang2, Wen-Kuan
} Huang ${ }^{1,7}$, Chiao-En Wu ${ }^{1,8}$, Jason Chia-Hsun Hsieh ${ }^{1,4}{ }^{\bowtie}$

1. Circulating Tumor Cell Lab, Division of Medical Oncology, Department of Internal Medicine, Chang Gung Memorial Hospital at Linkou, College of Medicine, Chang Gung University, Taoyuan, Taiwan;

2. Graduate Institute of Clinical Medical Sciences, College of Medicine, Chang Gung University, Taiwan;

3. Graduate Institute of Biochemical and Biomedical Engineering, Chang Gung University, Taoyuan City, Taiwan;

4. Department of Chemical and Materials Engineering, Chang Gung University, Taoyuan, Taiwan;

5. Division of Hematology-Oncology, Department of Internal Medicine, Chang Gung Memorial Hospital, Keelung and Chang Gung University, College of Medicine, Taiwan

6. Cancer Center, Chang Gung Memorial Hospital, Keelung, Taiwan.

7. Department of Oncology-Pathology, Karolinska Institutet, Stockholm, Sweden; Cancer Center Karolinska, Karolinska University Hospital, Stockholm SE-17176, Sweden

8. Northern Institute for Cancer Research, School of Medicine, Newcastle University, Newcastle, United Kingdom.

*Drs. Chou, $\mathrm{Wu}$ and Chang contributed equally to this work.

$\triangle$ Corresponding author: Dr. Jason Chia-Hsun Hsieh, MD. PhD. E-mail: wisdom2000@gmail.com; Tel.: +886-3-281200 ext. 2517

(c) Ivyspring International Publisher. This is an open access article distributed under the terms of the Creative Commons Attribution (CC BY-NC) license (https://creativecommons.org/licenses/by-nc/4.0/). See http://ivyspring.com/terms for full terms and conditions.

Received: 2017.10.05; Accepted: 2017.12.14; Published: 2018.01.12

\begin{abstract}
Background: There is an urgency to develop robust prognostic biomarkers for metastatic colorectal cancer $(\mathrm{mCRC})$ patients receiving chemotherapy. The current study aimed to examine the prognostic significance of circulating tumour cells (CTCs) and to develop a prognostic model incorporating CTCs in predicting the outcomes of $\mathrm{mCRC}$ patients treated with chemotherapy.

Methods: Our study prospectively enrolled $55 \mathrm{mCRC}$ patients who had undergone palliative chemotherapy between 2011 and 2014. Baseline CTCs and clinicopathological variables predictive of survival outcome were identified using univariate analysis. Negative selection-based protocol plus flow cytometry was used for CTC identification.

Results: The median overall survival (OS) and progression-free survival (PFS) were 24.2 months and 8.7 months, respectively. CTCs were detected in all the patients, and the median number of CTCs was 30.8/mL (range: $5.8-431.3 / \mathrm{mL}$ ). The median OS and PFS were 37.1 and 13.3 months, respectively, for patients with CTC number $\leq 30 / \mathrm{mL}$, while the median OS and PFS were 14.9 months and 5.1 months, respectively, for patients with CTC number $>30 / \mathrm{mL}$ (both $\mathrm{P}<0.001$ ). A prognostic model using CTCs in conjunction with other independent clinical variables further stratified patients into good and poor prognostic groups. The median OS and PFS were 32.4 and 11.5 months, respectively, in the good prognostic group and 5.4 and 2.7 months, respectively, in the poor prognostic group.

Conclusions: We developed a reliable CTC-based prognostic model for the prediction of clinical outcomes in $\mathrm{mCRC}$ patients treated with chemotherapy. This model may be used to assist clinicians in identifying those with the poorest prognosis before treatment.
\end{abstract}

Key words: metastatic colorectal cancer, circulating tumour cell, palliative chemotherapy, prognosis, model

\section{Introduction}

Colorectal cancer $(\mathrm{CRC})$ is one of the most common and lethal cancers, accounting for $10 \%$ of new cancer cases and $8.5 \%$ of total cancer-related deaths worldwide in 2012.[1] Systemic chemotherapy 
remains the most effective therapy for patients with unresected metastatic colorectal cancer (mCRC), and the median survival duration is now approaching 30 months for patients treated with cytotoxic chemotherapy and biologic agents targeting angiogenesis and the epidermal growth factor receptor.[2,3] Although the treatment response of these combinations has been fairly improved in the modern era, some patients who did not respond to these systemic treatments still have a dismal outcome. Indeed, approximately $28-44 \%$ of patients have no response to the first-line treatment with doublet chemotherapy plus either cetuximab or bevacizuamb.[2] Therefore, there is an urgency to develop robust prognostic biomarkers for mCRC patients receiving chemotherapy. Unfortunately, there has not yet been an accepted biologic or molecular marker of prognostic value for systemic chemotherapy.

In recent years, circulating tumour cells (CTCs) has been widely proposed to serve as biomarkers in various cancer types, including breast,[4, 5] prostate[6] and colorectal[7, 8] cancers. CTCs are cells migrating from solid tumours into the peripheral blood, which represent a disseminated disease and poor prognosis.[9-11] The CellSearch platform (Veridex, Raritan, NJ) is the only system approved by the US Food and Drug Administration to detect CTCs since 2004.[4] In 2008, Cohen et al first reported using the number of CTCs as a prognostic and predictive value for survival outcome and treatment response in mCRC patients treated with systemic chemotherapy. [7] However, the application of CTCs to predict the clinical outcomes of $\mathrm{mCRC}$ remains uncertain due to the relatively low efficiency of detection rate by the CellSearch platform[11] and no other device has been proven to be a standard method to date. In addition to CTCs, a number of clinical variables with predictive values have been identified in patients with mCRC receiving chemotherapy, including patient performance, [12] anatomic extent of the tumour,[13] histologic subtype,[14, 15] tumour grade,[16] serum tumour marker carcinoembryonic antigen (CEA),[17] and history of colectomy.[18] We hypothesized that a reliable prognostic tool combing clinicopathological features and CTCs for predicting the survival outcome and tumour response for mCRC patients would be most useful and possibly better to identify patients with the best and poorest prognoses rather than CTCs alone. To achieve this, the current study aimed to examine the prognostic significance of CTCs by using an easy-to-perform CTC detection platform using negative selection strategy and to develop a prognostic model that incorporates CTCs with other important clinical variables in predicting the survival outcome and tumour response in mCRC patients treated with systemic chemotherapy, especially to identify those patients with the poorest survival outcome to systemic chemotherapy.

\section{Methods}

\section{Patient selection and treatment of systemic chemotherapy}

This study was designed as a prospective observational study at a single institute. In total, fifty-five patients who had a histological diagnosis of CRC and were receiving systemic chemotherapy treatment at Chang Gung Memorial Hospital (CGMH) Linkou branch between January 2011 and June 2014 were prospectively enrolled. The eligibility criteria included patients over 20 years of age, patients with unresected metastatic disease, and patients with a measurable lesion confirmed by image studies. Patients who received concurrent radiotherapy or palliative tumour resection within 28 days of palliative chemotherapy and those who received local ablation therapy during the enrolment period were excluded. All patients underwent a baseline evaluation that included demographic data, clinical history, pathological characteristics, computed tomography (CT) scan, and biochemical evaluation. Systemic antitumour therapy consisted of a doublet combination of cytotoxic chemotherapy with or without bevacizumab or cetuximab (limited in patients with KRAS wild type), depending on the physician's decision. Tumour response was evaluated by CT scan according to the response evaluation criteria in solid tumours (RECIST) 1.1.[19] Image studies were performed and interpreted by an institutional radiologist at baseline and were repeated every 8-12 weeks (the exact interval was at the physician's discretion) to evaluate tumour response. The overall survival (OS) and progression-free survival (PFS) lengths were calculated from the date of administration of the first cycle of palliative chemotherapy to the date of death or disease progression after chemotherapy. All patients were followed-up until death or June 30, 2016. The study protocol was approved by the Institutional Review Board of CGMH (IRB number 101-2161C and 102-2407B). Written informed consent to participate was obtained from all the patients.

\section{Measurement of peripheral circulating tumour cells}

CTC analysis was performed using a protocol of combined negative selection and positive detection strategies, which was designed and validated in our previous reports.[20, 21] Briefly, the methods were (1) 
a negative selection protocol for effective $R B C$ and leukocyte depletion by red blood cell lysis and a CD45 depletion kit and (2) flow cytometry to quantitatively identify CTCs and calculate their numbers. The CTC identification was validated with a recovery rate of $44.6 \pm 9.1 \%$ and a coefficient of variation of $20.4 \%$.[20]

For CTC tests, $4 \mathrm{~mL}$ of peripheral blood was used after discarding the first $4 \mathrm{~mL}$ of blood to avoid epithelial contamination. Red blood cell lysis was performed within 72 hours after blood drawing. Samples were then negatively enriched by adding EasySep CD45 Depletion Cocktail (STEMCELL Technologies Inc., Vancouver, BC, Canada) at 25 $\mu \mathrm{L} / \mathrm{mL}$ to the cells and EasySep Magnetic Nanoparticles (STEMCELL) at $50 \mu \mathrm{L} / \mathrm{mL}$ to the cells. Immunomagnetically enriched samples containing spiked HCT116 cells were collected and labelled with Alexa Fluor ${ }^{\circledR}$ 488-conjugated anti-EpCAM monoantibody (1:400 dilution; Cell Signalling Technology, Inc., Danvers, MA, USA.) and a novel DNA-detecting far-red-fluorescing dye, Draq5 $(50 \mu \mathrm{M}$, Abcam, Cambridge, MA, USA), for nuclear staining. The isotype for EpCAM was also used for internal controls. In addition, we routinely used $4 \mathrm{~mL}$ of peripheral blood drawn from healthy individuals that was spiked with and without 1000 HCT116 cells (a colon cancer cell line, which was obtained from the Food Industry Research and Development Institute, Taiwan) for controls during the trial. The performance recovery, which was defined as the number of HCT116 cells detected by flow cytometry (BD FACSCalibur, BD Biosciences, San Jose, CA, USA) divided by the number of spiked HCT116 cells, and the coefficient of variation $(\mathrm{CV})$ value have been calculated and reported to be stable in a previous report.[20] CTCs were defined as the cells that were positive both for EpCAM and Draq5. Serum CEA levels of enrolled patients were measured by standard kit purchased from Siemens ADVIA Centaur Chemiluminescent Immunoassay, Siemens Siemens Healthcare GmbH, Germany.

\section{Statistical analysis}

The patients' demographic data were summarized as the number (\%) for categorical variables, and the median, 95\% confidence interval (CI), and range for continuous variables. Eleven predefined variables, included age, gender, primary tumor site, histological grade, American Joint Committee on Cancer (AJCC) staging group, Eastern Cooperative Oncology Group (ECOG) performance scale, KRAS mutation status, CEA level, CTC numbers, previous history of colectomy, and chemotherapy regimens, recorded within 7 days before the first cycle of palliative chemotherapy in mCRC patients, were then evaluated to ascertain their impact on OS and PFS. These potentially key prognostic variables were selected because minimal data were missing, and they could widely represent the available clinical data, which makes the findings broadly applicable. Variables with a $\mathrm{P}$ value $<0.10$ in the univariate analysis were included for analysis in the multivariate model. Multivariate Cox's proportional hazard model analysis was performed to investigate the impact of independent factors on OS and PFS. A risk model was developed from a multivariate logistic regression. The $\beta$-coefficients from the risk model were used to generate the points of the prognostic score for calculating survival time. Akaike's information criterion (AIC) was used to develop different multivariate models by systematically removing predictors from a full model that contained all predictive factors that were found to be statistically significant in the multivariate analysis. Receiver operating characteristic (ROC) curves and the area under the curve (c-statistic) for the outcome of survival at 1,2 , and 3 years were calculated to determine the accuracy of the prognostic score. Patients were further stratified into two prognostic groups according to their total score obtained from the prognostic score. Fisher's exact test was performed to determine significant associations between the tumour responses of the chemotherapy and prognostic groups. OS and PFS among different prognostic categories were calculated according to the Kaplan-Meier method. Log-rank tests were used to determine significant differences among the survival curves. SPSS 17.0 software (SPSS Inc., Chicago, IL) was used for all the statistical analyses. All statistical assessments were 2-sided. A P value of less than 0.05 was considered significant.

\section{Results}

Table 1 shows the demographic data of the 55 patients. The median age was 60 years (range: 36-88 years), and $61.8 \%$ of the patients were male. Forty-two patients $(76.4 \%)$ had a tumour that originated from the colon, and 13 patients $(23.6 \%)$ had tumours that originated from the rectum. Moderately differentiated adenocarcinoma was the most common histological differentiation subtype (38 patients, $69.1 \%$ ), followed by poorly (14 patients, $25.5 \%$ ) and well-differentiated (3 patients, 5.5\%) subtypes. Seventeen patients (30.9\%) had a mutated KRAS gene, and 23 patients (41.8\%) had previously undergone a colectomy for primary tumour resection. All the patients received 5-fluouracial based doublet chemotherapy. CTCs were detected in all the patients. The median number of CTCs was $30.8 / \mathrm{mL}$ (range: $5.8-431.3 / \mathrm{mL}$ ). There was no significant difference of CTC numbers among 
patients with different tumor stage and $\mathrm{T}$ - or $\mathrm{N}$ classification (Figure 1). Eighteen patients (32.7\%) had a normal baseline carcinoembryonic antigen (CEA) level ( $\leq 5 \mathrm{ng} / \mathrm{dL}$ ), and the median serum level of CEA was $10.5 \mathrm{ng} / \mathrm{dL}$ (range: $0.7-2558 \mathrm{ng} / \mathrm{dL}$ ) among all the patients.

Table 1. Patients' demographic data

\begin{tabular}{|c|c|c|c|}
\hline Variable & Category & no & $\%$ \\
\hline Age, year & Median (range) & $60(36-88)$ & \\
\hline \multirow[t]{2}{*}{ Gender } & male & 34 & 61.8 \\
\hline & female & 21 & 38.2 \\
\hline \multirow[t]{2}{*}{ Primary tumour site } & colon & 42 & 76.4 \\
\hline & rectum & 13 & 23.6 \\
\hline \multirow[t]{3}{*}{ Histological grade } & well & 3 & 5.5 \\
\hline & moderately & 38 & 69.1 \\
\hline & poorly & 14 & 25.5 \\
\hline \multirow[t]{2}{*}{ AJCC Stage, $7^{\text {th }}$ edition } & $4 a$ & 21 & 34.5 \\
\hline & $4 \mathrm{~b}$ & 34 & 65.5 \\
\hline \multirow[t]{3}{*}{ ECOG performance scale } & 0 & 11 & 20.0 \\
\hline & 1 & 25 & 45.5 \\
\hline & $2 \sim 3$ & 19 & 34.5 \\
\hline \multirow[t]{2}{*}{ KRAS mutation status } & no mutation & 38 & 69.1 \\
\hline & mutation & 17 & 30.9 \\
\hline \multirow[t]{3}{*}{ CEA, ng/dL } & median (range) & $10.5(0.7-2558)$ & \\
\hline & $\leq 5$ & 18 & 32.6 \\
\hline & $>5$ & 37 & 67.4 \\
\hline CTC, number $/ \mathrm{mL}$ & median (range) & $36.8(5.8-431.3)$ & \\
\hline \multirow{2}{*}{ Previous history of colectomy } & no & 32 & 58.2 \\
\hline & yes & 23 & 41.8 \\
\hline \multirow[t]{3}{*}{ Line of chemotherapy } & 1 & 53 & 96.4 \\
\hline & 2 & 1 & 1.8 \\
\hline & 3 & 1 & 1.8 \\
\hline \multirow[t]{5}{*}{ Chemotherapy regimen } & Fluorouracil & 55 & 100 \\
\hline & Irinotecan & 39 & 70.9 \\
\hline & Oxaliplatin & 16 & 29.1 \\
\hline & Bevacizumab & 34 & 61.8 \\
\hline & Cetuximab & 7 & 12.7 \\
\hline
\end{tabular}

AJCC: American Joint Committee on Cancer; ECOG: Eastern Cooperative Oncology Group

CEA: carcinoembryonic antigen; CTC: circulating tumour cell

At the study end, 42 patients $(76.4 \%)$ died during the study. The median OS and PFS were 24.2 months (95\% CI: 14.0-34.4 months) and 8.7 months (95\% CI: 6.1-11.3 months), respectively. The 1-, 2-, and 3-year survival rates were $74.5 \%, 50.2 \%$, and $31.9 \%$, respectively. The results of the univariate analyses for OS and PFS that were associated with the clinical variables are presented in Table 2 . Based on the univariate analysis of the imputed data set, 6 of the 11 preselected variables, including primary tumour side, tumour stage, histological grade, previous history of colectomy, ECOG performance scale, and CTCs, showed a statistically significant effect on OS. However, the multivariate analysis identified tumour stage, histological grade, previous history of colectomy, ECOG performance score, and number of
CTCs as the only independent prognostic factors (Figure 2). Regarding the PFS of all the patients, 3 of the 11 preselected variables, including previous history of colectomy, ECOG performance scale and number of CTCs, were significant variables in both the univariate analysis and multivariate analysis. Using the median number of CTCs as the cut-off point, the median OS and PFS were 37.1 months (95\% CI: 22.6-51.6 months) and 13.3 months (95\% CI: 11.7-14.9 months), respectively, for patients with CTC number $\leq 30 / \mathrm{mL}$, while the median OS and PFS were 14.9 months (95\% CI: 8.6-21.2 months) and 5.1 months (95\% CI: 3.5-6.7 months), respectively, for patients with CTC number $>30 / \mathrm{mL}$ (both $\mathrm{P}<0.001$, Figure 3).

The risk model and scoring system of the prognostic score generated from $\beta$-coefficients of multivariate analysis for OS are shown in Table 3. The total prognostic scores ranged from 0 to 6 . Using the prognostic score, patients were stratified into good (total score of 0-3) and poor (total score of 4-6) prognostic groups. This prognostic scoring system assigned $70.9 \%$ of the patients to the good prognostic group and $29.1 \%$ of the patients to the poor prognostic group. The median $\mathrm{OS}$ in the good and poor prognostic risk groups were 32.4 months (95\% CI: 23.0-41.8 months) and 5.4 months (95\% CI: 2.1-8.7 months), respectively (Figure 4A). The hazard ratio was 10.3 (95\% CI: 3.4-23.5; P < 0.001) when comparing the poor and good prognostic groups. Accordingly, the median PFS in the good and poor prognostic risk groups was 11.5 months (range: 8.4-14.6 months) and 2.7 months (range: 1.3-4.1 months), respectively, with a hazard ratio of 5.15 (95\% CI: 2.68-9.57; P < 0.001) (Figure 4B). The c-statistic that was obtained using ROC curve analysis for mortality within 1, 2, and 3 years was 0.82 (95\% CI: 0.71-0.94), 0.83 (95\% CI: $0.72-0.93$ ), and 0.72 (95\% CI: 0.58-0.86), respectively. The AIC was 230.6 for the full model and 257.3 after removal of the variable of CTCs from the full model. A smaller AIC value indicated a higher capacity for predictive stratification in the prognostic model. Table 4 compared the performance of CTC numbers, clinical parameter scoring system except CTC and combined CTC numbers and clinical parameter scoring system (full score) for OS and PFS. The results showed the full score and CTC number performed well both for PFS and OS while clinical parameters did not. Therefore, our findings suggest CTC alone could have good performance for outcome prediction, but better performance was found when combined with clinical parameters. 
Table 2. Univariate analyses for overall survival (OS) and progression-free survival (PFS)

\begin{tabular}{|c|c|c|c|c|c|c|c|c|c|c|}
\hline \multirow[b]{2}{*}{ Variable } & \multirow[b]{2}{*}{ Category } & \multirow[b]{2}{*}{ No (\%) } & \multicolumn{4}{|c|}{ Univariate for OS } & \multicolumn{4}{|c|}{ Univariate for PFS } \\
\hline & & & No of event (\%) & median & $95 \% \mathrm{CI}$ & $\mathrm{p}$ & $\begin{array}{l}\text { No of } \\
\text { event } \\
(\%)\end{array}$ & median & $95 \% \mathrm{CI}$ & $\mathrm{p}$ \\
\hline Overall & & $55(100)$ & $42(76.4)$ & 24.2 & $14.0-34.4$ & & $54(98.2)$ & 8.7 & $6.1-11.3$ & \\
\hline \multirow[t]{2}{*}{ Age } & $\leq 60$ & $28(50.9)$ & $19(67.9)$ & 26.2 & $17.8-34.6$ & 0.13 & $27(96.4)$ & 9.1 & $6.4-11.8$ & 0.34 \\
\hline & $>60$ & $27(49.1)$ & $23(85.2)$ & 19.8 & $4.4-35.2$ & & $27(100)$ & 7.5 & $5.5-9.5$ & \\
\hline \multirow[t]{2}{*}{ Gender } & male & $34(61.8))$ & $25(73.5)$ & 25.1 & $16.9-33.3$ & 0.6 & $33(97.1)$ & 8.7 & $5.7-11.7$ & 0.46 \\
\hline & female & $21(38.2)$ & $17(81.0)$ & 19.8 & $8.6-30.9$ & & $21(100)$ & 7.5 & $4.7-10.3$ & \\
\hline \multirow[t]{2}{*}{ Primary site } & colon & $42(76.4)$ & $29(69.0)$ & 26.2 & $19.8-32.6$ & 0.047 & $41(97.6)$ & 8.2 & $5.6-10.8$ & 0.49 \\
\hline & rectum & $13(33.6)$ & $13(100)$ & 15.7 & $10.1-21.3$ & & $13(100)$ & 9.7 & $2.3-17.1$ & \\
\hline \multirow[t]{2}{*}{ AJCC stage, $7^{\text {th }}$} & IVa & $21(38.2)$ & $14(66.7)$ & 34.6 & $22.6-46.4$ & 0.026 & $20(95.2)$ & 12.9 & $6.8-19.0$ & 0.17 \\
\hline & $\mathrm{IVb}$ & $34(61.8)$ & $28(82.4)$ & 15.7 & $11.4-20.0$ & & $34(100)$ & 7.8 & $6.8-8.8$ & \\
\hline \multirow[t]{2}{*}{ Grade } & well or moderately & $41(74.5)$ & $31(75.6)$ & 26.3 & $20.3-32.3$ & 0.008 & $40(97.6)$ & 9.1 & $6.5-11.7$ & 0.69 \\
\hline & poorly & $14(25.5)$ & $11(78.6)$ & 12.5 & $4.2-20.8$ & & $14(100)$ & 7.5 & $6.6-8.4$ & \\
\hline \multirow{3}{*}{$\begin{array}{l}\text { Previous history of } \\
\text { colectomy }\end{array}$} & yes & $23(41.8)$ & $12(52.2)$ & 43.9 & $12.9-74.9$ & $<0.001$ & $22(95.7)$ & 12.8 & $7.9-17.7$ & 0.022 \\
\hline & no & $32(58.2)$ & & 15.7 & $9.6-21.8$ & & & 7.2 & $4.9-9.5$ & \\
\hline & & & $30(93.8)$ & & & & $32(100)$ & & & \\
\hline \multirow[t]{2}{*}{ KRAS mutation } & mutant & 17 (30.9) & $14(82.4)$ & 17.4 & $7.0-27.8$ & 0.27 & $17(100)$ & 7.2 & $1.7-12.7$ & 0.63 \\
\hline & no mutant & $38(69.1)$ & $28(73.7)$ & 27.7 & $18.9-36.6$ & & $37(97.4)$ & 9.7 & $6.8-12.6$ & \\
\hline \multirow[t]{2}{*}{ Chemotherapy regimen } & Oxaliplatin-based & $17(30.9)$ & $13(76.5)$ & 15.7 & $8.6-22.8$ & 0.48 & $17(100)$ & 5.4 & $0.0-9.8$ & 0.14 \\
\hline & Irinotecan-based & $38(69.1)$ & $29(76.3)$ & 26.3 & $17.6-34.9$ & & $37(97.4)$ & 10.6 & $8.3-12.9$ & \\
\hline \multirow[t]{2}{*}{ ECOG performance } & $0 \sim 1$ & $36(65.5)$ & $24(66.7)$ & 31.8 & $21.0-42.6$ & $<0.001$ & $35(97.2)$ & 11.5 & $8.0-15.0$ & $<0.001$ \\
\hline & $2 \sim 4$ & $19(45.5)$ & $18(94.7)$ & 10.8 & $1.7-19.9$ & & $19(100)$ & 3.8 & $2.1-5.5$ & \\
\hline \multirow[t]{2}{*}{ CEA level, ng/dL } & $\leq 5$ & $18(32.7)$ & $13(72.2)$ & 25.1 & $14.0-36.2$ & 0.92 & $17(94.4)$ & 7.4 & $2.0-12.8$ & 0.57 \\
\hline & $>5$ & $37(67.3)$ & $29(78.4)$ & 21.6 & $9.2-34.0$ & & 37 (100) & 10 & $6.7-13.3$ & \\
\hline \multirow{2}{*}{$\begin{array}{l}\text { CTC, numbers/ } \\
\text { mL }\end{array}$} & $\leq 30$ & $26(47.3)$ & $17(65.4)$ & 37.1 & $22.5-51.6$ & 0.005 & $25(96.2))$ & 13.3 & $11.7-14.9$ & $<0.001$ \\
\hline & $>30$ & $29(52.7)$ & $25(86.2)$ & 14.9 & $8.9-21.2$ & & $29(100)$ & 5.1 & $3.5-6.7$ & \\
\hline
\end{tabular}

AJCC: American Joint Committee on Cancer; ECOG: Eastern Cooperative Oncology Group

CEA: carcinoembryonic antigen; CTC: circulating tumour cell

Table 3. Point scoring of overall survival for the prognostic models

\begin{tabular}{lllc}
\hline Variable & Point & & \\
\cline { 2 - 3 } & 0 & IVb & \\
\hline AJCC 7th stage & IVa & $>30$ & \\
CTC number, per mL & $\leq 30$ & & $2-3$ \\
ECOG performance & $0-1$ & poorly & \\
Histological grade & well or moderately & no \\
Previous history of colectomy & yes & \\
\hline \multicolumn{2}{l}{ AJCC: American Joint Committee on Cancer; CTC: circulating tumour cell; ECOG: } \\
Eastern Cooperative Oncology Group
\end{tabular}

Overall, chemotherapy resulted in tumours showing a partial response in $26(47.3 \%)$ patients, stable disease in $13(23.6 \%)$ patients, and progressive disease in the remaining $16(29.1 \%)$ patients. The treatment response to a partial response, stable disease, and progressive disease of tumours were 25 $(64.1 \%)$ patients, $11(28.2 \%)$ patients, and $3(7.7 \%)$ patients, respectively, in patients from the good risk group, while the treatment responses to a partial response, stable disease, and progressive disease of tumours were $1(6.3 \%)$ patient, $2(12.5 \%)$ patients, and $16(81.3 \%)$ patients, respectively, in the poor risk group (Fisher's exact test $P<0.001$, Figure 5). Figure 6 presents the correlations between Kras mutation status and CTC numbers (6a) as well as targeted agents and CTC numbers (6b). There was no significant difference of CTC numbers between patients with Kras mutant and wild type (Mann-Whitney $\mathrm{U}$ test $p=0.879$ ) as well as CTC numbers between patients received bevacizumabbased for cetuximab-based chemotherapies (MannWhitney $\mathrm{U}$ test $p=0.933$ ).

Table 4. The performance of CTC numbers, clinical parameter scoring system except CTC and combined CTC numbers and clinical parameter scoring system (full score) for OS and PFS

\begin{tabular}{lll}
\hline & Hazard Ratio (95\% CI) & $p$ value \\
\hline For OS & & \\
CTC alone (> 30 vs $\leq \mathbf{3 0}$ cells/mL) & $2.613(1.385-4.928)$ & 0.003 \\
Clinical parameter scoring except CTC & $3.266(1.715-6.219)$ & $<0.001$ \\
Full Score & $6.135(1.867-20.161)$ & 0.003 \\
For PFS & & \\
CTC alone (> 30 vs $\leq \mathbf{3 0}$ cells/mL) & $4.942(2.600-9.392)$ & $<0.001$ \\
Clinical parameter scoring except CTC & $1.830(1.041-3.217)$ & 0.036 \\
Full Score & $3.741(1.631-8.581)$ & 0.002 \\
\hline
\end{tabular}


A

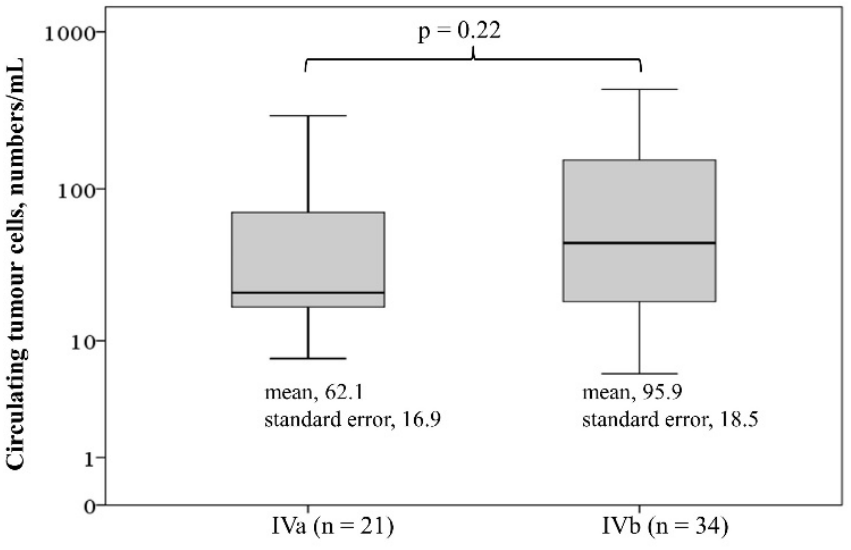

AJCC stage, 7th edition

\section{B}
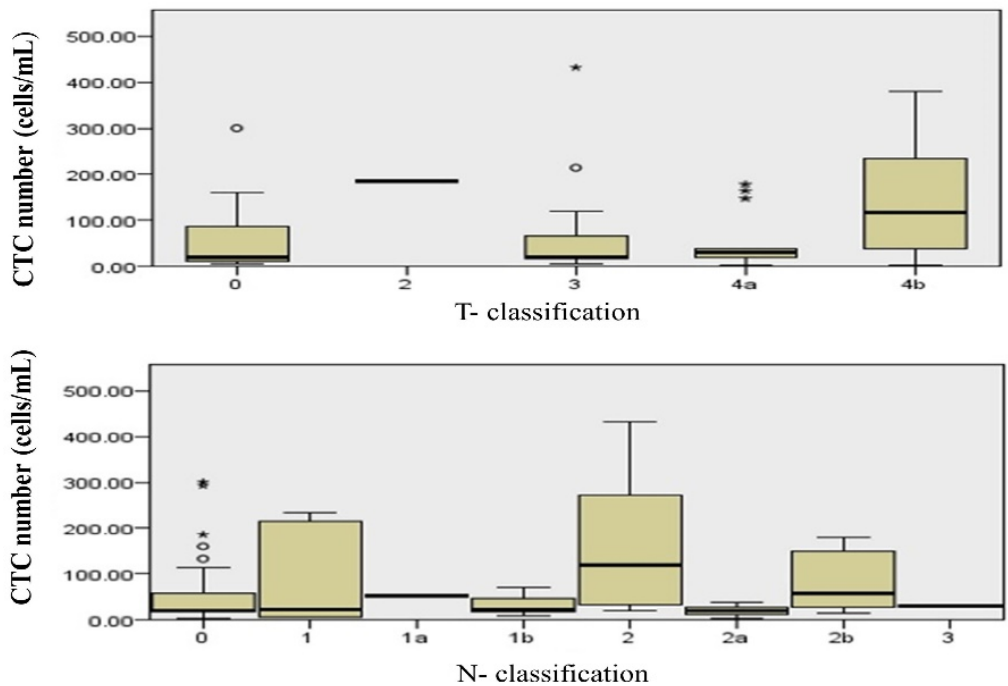

Figure 1. Difference of CTC numbers among different tumor stage (1A), and T- or N- classification (Figure 1B)

Variable

\section{Overall survival}

stage IVb vs. IVa

CTC $>30$ vs. $\leq 30$, numbers $/ \mathrm{mL}$

ECOG $2-3$ vs. $0-1$

Tumor grade Poorly vs. Moderately/Well

Colectomy history Yes vs. No

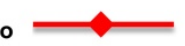

Progression-free survival

CTC $>30$ vs. $<30$, numbers $/ \mathrm{mL}$

ECOG $2-3$ vs. $0-1$

Colectomy history Yes vs. No

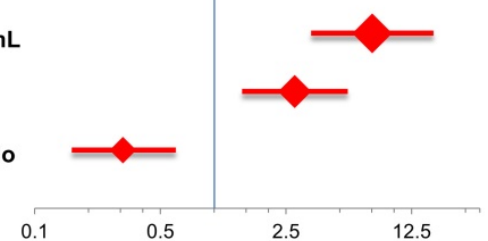

Hazard Ratio $(95 \% \mathrm{Cl})$

$3.20(1.41-7.26)$

$2.47(1.07-5.71)$

$6.71(2.68-16.8)$

$3.39(1.47-7.78)$

$0.24(0.09-0.60)$

$7.56(3.45-16.6)$

$2.80(1.43-5.51)$

$0.31(0.16-0.61)$

Figure 2. Multivariate analysis for overall survival and progression-free survival. 
A

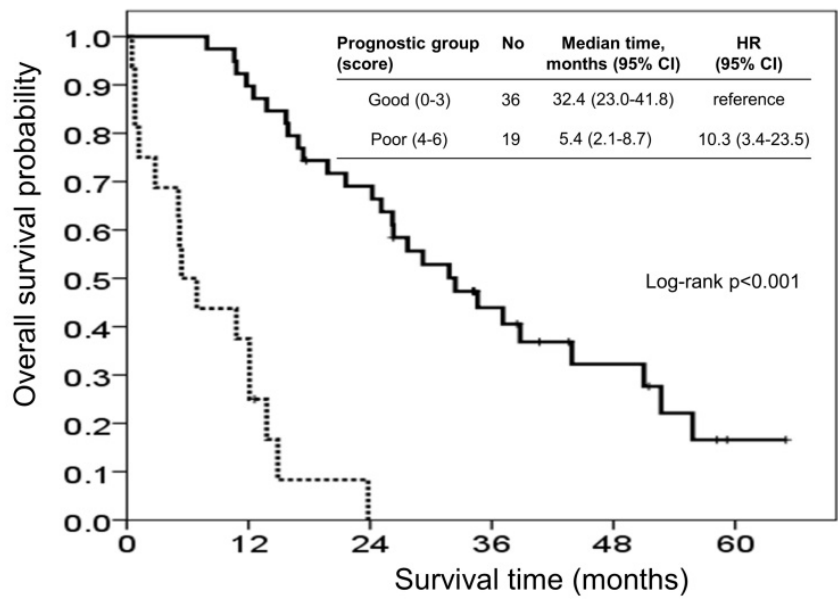

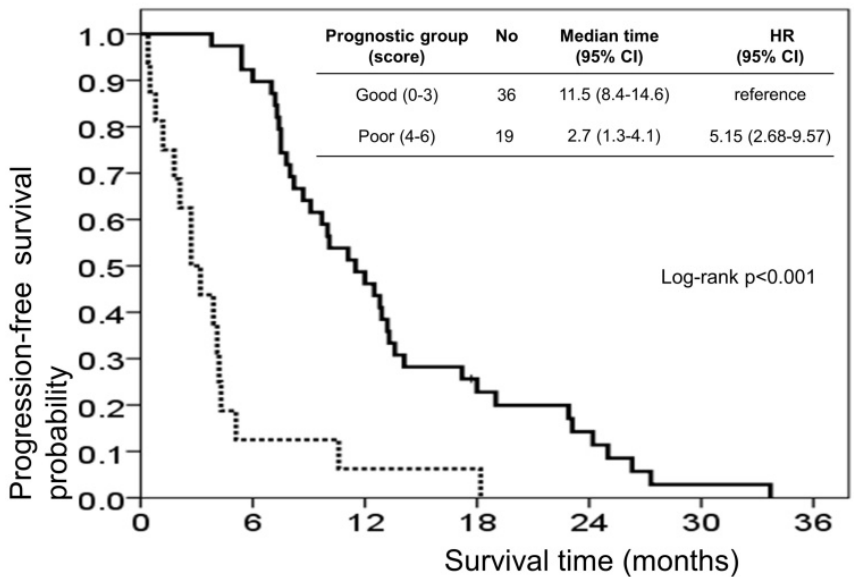

Figure 3. Kaplan-Meier overall survival (A) and progression-free survival (B) curves for patients, stratified according to the number of circulating tumour cells.

A

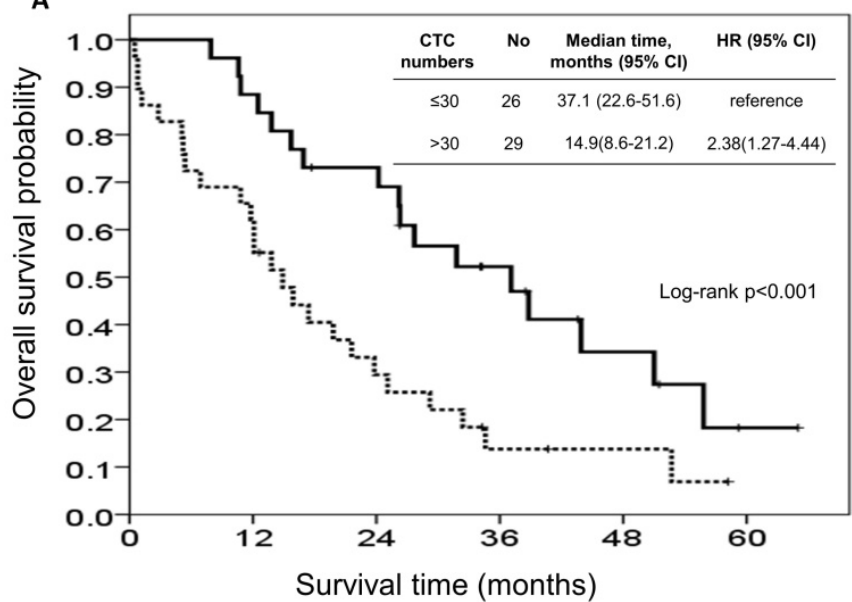

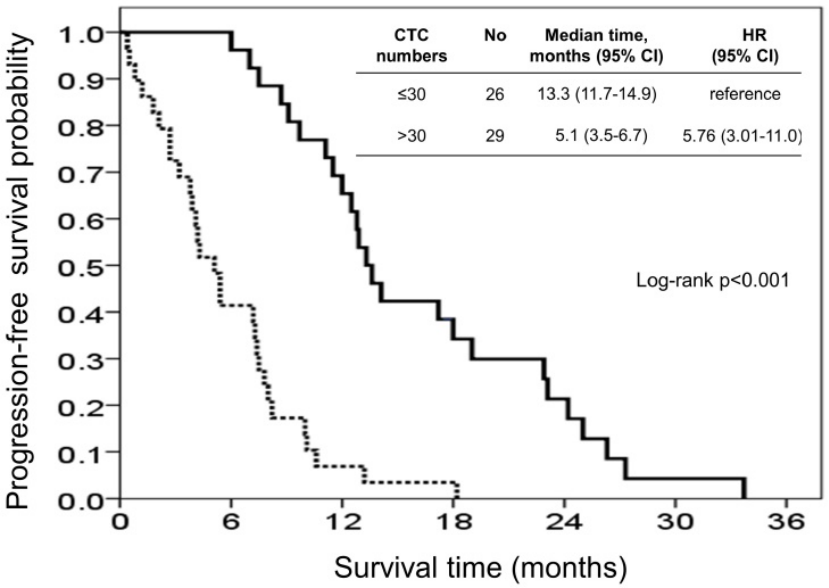

Figure 4. Kaplan-Meier overall survival (A) and progression-free survival (B) curves for patients, stratified according to the prognostic model.

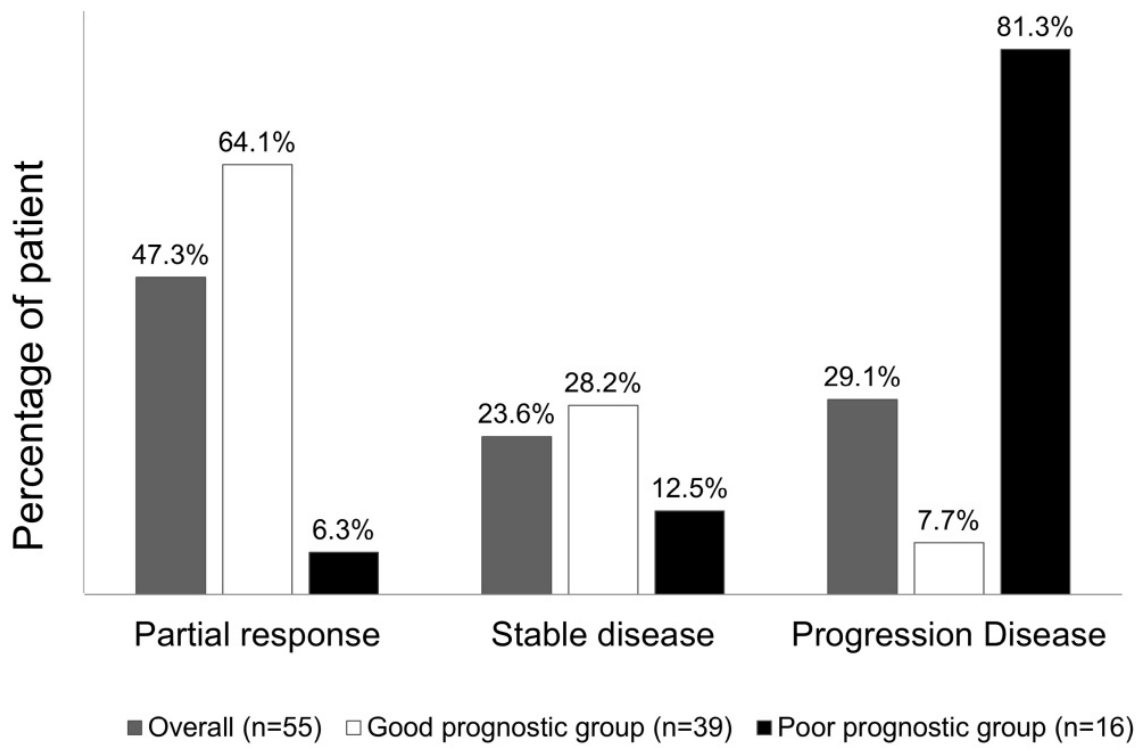

Figure 5. Tumour response rate to chemotherapy, stratified according to the prognostic model. 


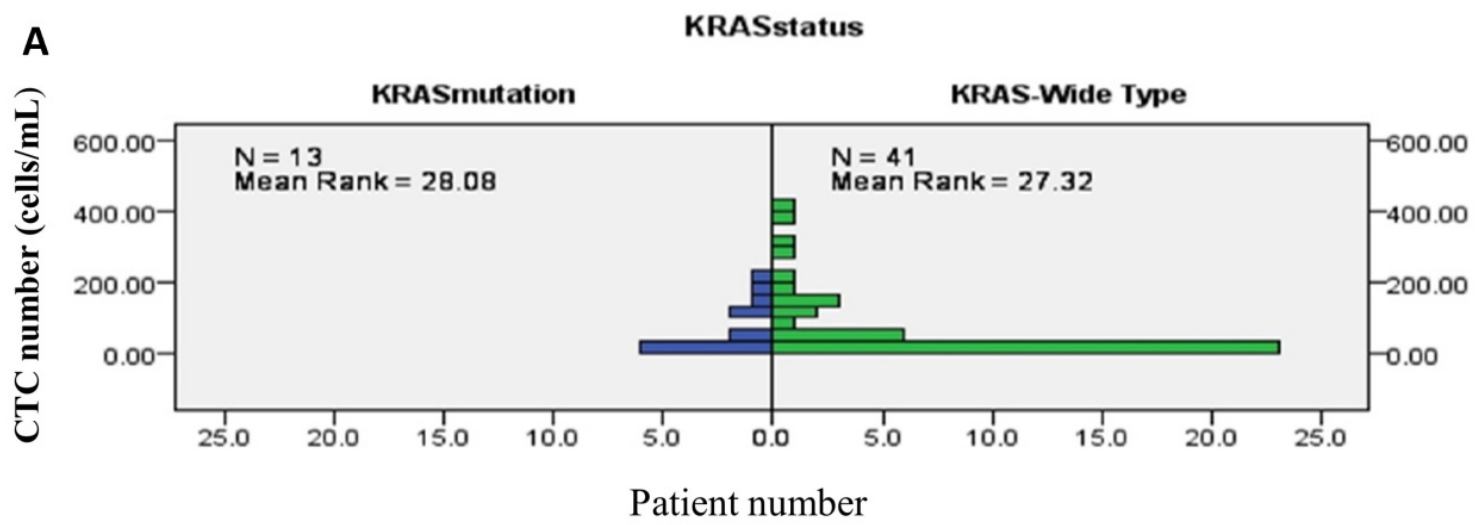

B

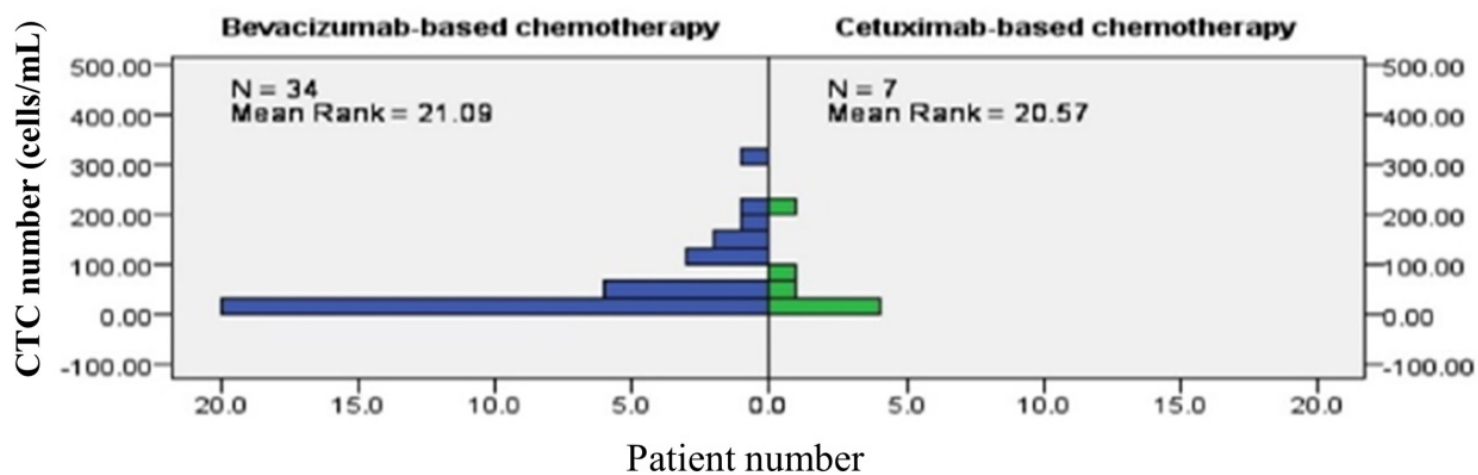

Figure 6 presents the correlations between Kras mutation status and CTC numbers $(6 A)$ as well as targeted agents and CTC numbers (6B).

\section{Discussion}

$\mathrm{mCRC}$ is a common and lethal malignancy both in Taiwan and worldwide. ${ }^{1,}$ [22] Though the survival outcome and treatment response to chemotherapy plus a biologic agent had dramatic advances in the modern era, a small subset of patients still had a dismal outcome, which implies a stubbornness to antitumour treatment.[2, 3] Therefore, a prognostic model for picking up on those with the poorest outcomes in antitumour therapy may assist clinicians and patients in decision making. Our study developed a prognostic model that predicts survival outcome in patients with mCRCs, using CTCs in conjunction with other significant clinical variables. This model accurately predicted survival outcome with c-indices of $0.824,0.828$, and 0.719 for the probability of 1-, 2-, and 3-year mortalities. Our study showed that this model might be informative for clinicians and patients with mCRCs for estimating the survival outcome after systemic chemotherapy.

Tumour stage ${ }^{13}$, histological grade[16], previous colectomy[18], and ECOG scale[12] are the well-established prognostic factors in patients with mCRC. Advanced stage and poor histological grade represented aggressive tumour behaviour. A population-based study including 37,793 mCRC patients recently reported that palliative primary tumour resection was associated with improved overall and cancer-specific survival.[18] A lower ECOG scale represented a better ability of daily activity and general health status[23, 24], which were associated with the eligibility to receive chemotherapy and a lower incidence of treatment-related toxicity. These are all possible reasons for explaining why the ECOG scale contributed the most in our model. Our study identified that the baseline number of CTCs prior to chemotherapy was an independent prognostic factor for overall survival and a predictive factor for PFS and tumour response in mCRC patients. Furthermore, conjunction of the CTCs with other prognostic clinical variables increased the discrimination power of the model (AIC value decreased from 257.3 to 230.6 after the CTC variable was added into the full model). Our result encourages integrating CTCs and clinical variables for predicting the outcome of mCRC patients receiving systemic chemotherapy. Patients with a good prognosis may be encouraged to receive palliative chemotherapy to gain the maximal benefit of the treatment. In contrast, palliative chemotherapy may be futile in patients with a poor prognosis, given that the median OS, PFS, and tumour response of these patients were only 5.4 months, 2.7 months and $6.3 \%$, respectively. 
The number of CTCs is an emerging biomarker that has been shown to be an independent prognostic factor in numerous studies of various solid cancers and CRC.[4-7] In general, higher CTC counts have been consistently associated with disseminated stage and aggressive tumour biology.[10, 11, 25] Cohen et al first reported that patients with CTC number $\geq 3$ per $7.5 \mathrm{~mL}$ of peripheral blood had a shorter median survival based on a prospective multicentre study of $430 \mathrm{mCRC}$ patients.[7] In their report, the distribution of the number of CTCs per $7.5 \mathrm{~mL}$ of peripheral blood using the CellSearch system as undetectable, 1 to 2, and 3 or more were $48 \%, 26 \%$, and $26 \%$, respectively. Furthermore, two large-scale meta-analysis studies, which included 3094 patients in the first study and 1329 patients in the second study, showed that the detection of CTCs correlated with a poor prognosis for CRC patients.[26, 27] In addition, the optimal cut-off value of the number of CTCs for the prognostic value in patients positive for CTCs was undetermined [28]. Furthermore, only approximately one-third of the patients had detectable CTCs, and the discrimination of survival differences among those patients who were negative for CTCs was impossible. Therefore, it is imperative that new techniques be developed for CTC enrichment and identification in order for a wide utilization of CTCs in MCRC. This study used a negative selection method of CD45 depletion using magnetic beads followed by flow cytometry for CTC identification.[20, 21] CTCs were detected in all patients with $\mathrm{MCRC}$, and the cut-off value of the median number of CTCs was analysed as an independent prognostic factor. Based on this simple and cheap method, we believed that CTCs may be widely available to use as a prognostic and predictive factor in $\mathrm{mCRC}$ patients who underwent palliative chemotherapy.

To the best of our knowledge, this is the largest prospective study using a negative selection method for CTC isolation to evaluate the prognostic role of CTCs in patients with mCRC. Based on this simple method, the clinical variables of the prognostic model are accessible and available before palliative chemotherapy. Therefore, the current model is widely applicable and clinically relevant to identifying a small subset of patients with the poorest outcome. However, there are several inherent limitations of this study. First, this study included small numbers of patients, selected from a single medical centre; therefore, the statistically significant impact of clinical variables on survival outcome might be limited due to the small sample size. Second, the chemotherapy regimen was not consistent in all patients, so analysis of the baseline CTCs relevant to PFS and tumour response might be confounded by different treatment regimens. Finally, and most importantly, even though our prognostic model represented a good accuracy to predict 1-, 2-, and 3-year survival outcomes by the c-index, an external validation of the model is essential before it can be widely used. A prospective study is required to evaluate the prognostic and predictive values of this model in mCRC patients who underwent palliative chemotherapy.

In conclusion, we identified the number of CTCs at the baseline of palliative chemotherapy as an independent prognostic factor for predicting survival outcome, in terms of overall survival, progression-free survival, and tumour response, in patients with mCRC. In addition, we developed a reliable CTC-based prognostic model for the prediction of survival outcome and treatment response in mCRC patients, after systemic chemotherapy. This model may be used to assist clinicians in identifying those mCRC patients with the poorest prognosis and treatment outcome. Further large-scale prospective trials to confirm the role of CTC prognosis score guiding anticancer strategies for each $\mathrm{mCRC}$ patient were eagerly warranted.

\section{Acknowledgements}

Funding for this study was received from the Chang Gung Memorial Hospital at Linkou (CMRPG3C1561 to Professor Wen-Chi Chou; CMRPG3E0512, CMRPG3G0591 and MOST 105-2314B-182A- 030- to Dr. Chia-Hsun Hsieh; CMRPD 2D0041-43 and CMRPD2E0011-1 to Professor Min-Hsien $\mathrm{Wu}$ ). These funding sources were used in the designing and conducting of the study: they were used for collecting, managing, analysing, and interpreting the data, and preparing, reviewing, or approving the article. All authors declare no financial or other conflicts of interest.

\section{Competing Interests}

The authors have declared that no competing interest exists.

\section{References}

1. GLOBOCAN 2012: estimated cancer incidence, mortality and prevalence worldwide in 2012. http://globocan.iarc.fr/Pages/fact_sheets_cancer.aspx. Accessed March 07, 2017.

2. Heinemann V, von Weikersthal LF, Decker T, Kiani A, Vehling-Kaiser U, Al-Batran S-E, Heintges T, Lerchenmüller C, Kahl C, Seipelt G. FOLFIRI plus cetuximab versus FOLFIRI plus bevacizumab as first-line treatment for patients with metastatic colorectal cancer (FIRE-3): a randomised, open-label, phase 3 trial. The lancet oncology. 2014; 15:1065-75. doi: 10.1016/S1470-2045(14)70330-4.

3. Lenz H, Niedzwiecki D, Innocenti F, Blanke C, Mahony M, O'Neil BH, Shaw JE, Polite B, Hochster H, Atkins J. 501ocalgb/Swog 80405: phase III trial of irinotecan/5-Fu/leucovorin (folfiri) or oxaliplatin/5-Fu/leucovorin (mfolfox6) with bevacizumab (bv) or cetuximab (cet) for patients (pts) with expanded Ras analyses untreated metastatic adenocarcinoma of the colon or rectum (mcrc). Annals of Oncology. 2014; 25:mdu438.13. doi: 10.1093/annonc/mdu438.13.

4. Cristofanilli M, Budd GT, Ellis MJ, Stopeck A, Matera J, Miller MC, Reuben JM, Doyle GV, Allard WJ, Terstappen LW, Hayes DF. Circulating tumor cells, 
disease progression, and survival in metastatic breast cancer. N Engl J Med. 2004; 351: 781-91. doi: 10.1056/NEJMoa040766.

5. Riethdorf S, Fritsche H, Muller V, Rau T, Schindlbeck C, Rack B, Janni W, Coith C, Beck K, Janicke F, Jackson S, Gornet T, Cristofanilli M, et al. Detection of circulating tumor cells in peripheral blood of patients with metastatic breast cancer: a validation study of the CellSearch system. Clin Cancer Res. 2007; 13: 920-8. doi: 10.1158/1078-0432.CCR-06-1695.

6. de Bono JS, Scher HI, Montgomery RB, Parker C, Miller MC, Tissing H, Doyle GV, Terstappen LW, Pienta KJ, Raghavan D. Circulating tumor cells predict survival benefit from treatment in metastatic castration-resistant prostate cancer. Clin Cancer Res. 2008; 14: 6302-9. doi: 10.1158/1078-0432.CCR-08-0872.

7. Cohen SJ, Punt CJ, Iannotti N, Saidman BH, Sabbath KD, Gabrail NY, Picus J, Morse M, Mitchell E, Miller MC. Relationship of circulating tumor cells to tumor response, progression-free survival, and overall survival in patients with metastatic colorectal cancer. Journal of clinical oncology. 2008; 26: 3213-21. doi: 10.1200/JCO.2007.15.8923.

8. Lu YJ, Wang P, Peng J, Wang X, Zhu YW, Shen N. Meta-analysis Reveals the Prognostic Value of Circulating Tumour Cells Detected in the Peripheral Blood in Patients with Non-Metastatic Colorectal Cancer. Sci Rep. 2017; 7: 905. doi: 10.1038/s41598-017-01066-y.

9. Pantel K, Brakenhoff $\mathrm{RH}$, Brandt B. Detection, clinical relevance and specific biological properties of disseminating tumour cells. Nat Rev Cancer. 2008; 8: 329-40. doi: $10.1038 / \mathrm{nrc} 2375$.

10. Sergeant G, Penninckx F, Topal B. Quantitative RT-PCR detection of colorectal tumor cells in peripheral blood-a systematic review. Journal of Surgical Research. 2008; 150: 144-52. doi: 10.1016/j.jss.2008.02.012.

11. Hardingham JE, Grover P, Winter M, Hewett PJ, Price TJ, Thierry B. Detection and clinical significance of circulating tumor cells in colorectal cancer-20 years of progress. Molecular Medicine. 2015; 21: S25. doi: 10.2119/molmed.2015.00149.

12. Chen D, Wen X, Song YS, Rhee YY, Lee TH, Cho NY, Han SW, Kim TY, Kang GH. Associations and prognostic implications of Eastern Cooperative Oncology Group performance status and tumoral LINE-1 methylation status in stage III colon cancer patients. Clin Epigenetics. 2016; 8: 36. doi: 10.1186/s13148-016-0203-8

13. Edge SB, Byrd DR, Compton CC, et al. AJCC (American Joint Committee on Cancer) Cancer Staging Manual, 7th ed. Springer, New York 2010; p.133.

14. Knox RD, Luey N, Sioson L, Kedziora A, Clarkson A, Watson N, Toon CW, Cussigh C, Pincott S, Pillinger S, Salama Y, Evans J, Percy J, et al. Medullary colorectal carcinoma revisited: a clinical and pathological study of 102 cases. Ann Surg Oncol. 2015; 22: 2988-96. doi: 10.1245/s10434-014-4355-5.

15. Pande R, Sunga A, Levea C, Wilding GE, Bshara W, Reid M, Fakih MG. Significance of signet-ring cells in patients with colorectal cancer. Dis Colon Rectum. 2008; 51: 50-5. doi: 10.1007/s10350-007-9073-7.

16. Newland RC, Dent OF, Lyttle MN, Chapuis PH, Bokey EL Pathologic determinants of survival associated with colorectal cancer with lymph node metastases. A multivariate analysis of 579 patients. Cancer. 1994; 73: 2076-82. doi: 10.1002/1097-0142(19940415)73:8<2076:AID-CNCR2820730811>3.0.CO; 2-6.

17. Thirunavukarasu P, Sukumar S, Sathaiah M, Mahan M, Pragatheeshwar KD, Pingpank JF, Zeh H, 3rd, Bartels CJ, Lee KK, Bartlett DL. C-stage in colon cancer: implications of carcinoembryonic antigen biomarker in staging, prognosis, and management. J Natl Cancer Inst. 2011; 103: 689-97. doi: 10.1093/jnci/djr078.

18. Tarantino I, Warschkow $\mathrm{R}$, Worni M, Cerny $\mathrm{T}$, Ulrich A, Schmied BM, Güller U. Prognostic Relevance of Palliative Primary Tumor Removal in 37,793 Metastatic Colorectal Cancer Patients: A Population-based, Propensity Score-adjusted Trend Analysis. Annals of surgery. 2015; 262: 112-20. doi: 10.1097/SLA.0000000000000860.

19. Eisenhauer E, Therasse P, Bogaerts J, Schwartz L, Sargent D, Ford R, Dancey J, Arbuck S, Gwyther S, Mooney M. 32 INVITED New response evaluation criteria in solid tumors: revised RECIST guideline version 1.1. European Journal of Cancer Supplements. 2008; 6: 13. doi: 10.1016/j.ejca.2008.10.026.

20. Su PJ, Wu MH, Wang HM, Lee CL, Huang WK, Wu CE, Chang HK, Chao YK, Tseng CK, Chiu TK, Lin NM, Ye SR, Lee JY, et al. Circulating Tumour Cells as an Independent Prognostic Factor in Patients with Advanced Oesophageal Squamous Cell Carcinoma Undergoing Chemoradiotherapy. Sci Rep. 2016; 6: 31423. doi: 10.1038 / srep31423.

21. Chiu T-K, Chou W-P, Huang S-B, Wang H-M, Lin Y-C, Hsieh C-H, Wu M-H Application of optically-induced-dielectrophoresis in microfluidic system for purification of circulating tumour cells for gene expression analysis-Cancer cell line model. Scientific Reports. 2016; 6. doi: 10.1038/srep32851.

22. Health registry annual Report 2013, Republic of China. Republic of China: Republic of China: Bureau of Health Promotion, Department of Health, Executive Yuan; 2015. Available at: http://www.hpa.gov.tw/BHPNet/ Web/Stat/Statistics.aspx. Accessed January 20, 2017.

23. Chou WC, Yeh KY, Peng MT, Chen JS, Wang HM, Lin YC, Liu CT, Chang PH, Wang CH, Chen PT, Hung YS, Lu CH, Li SH. Development and Validation of a Prognostic Score to Predict Survival in Adult Patients With Solid Tumors and Bone Marrow Metastases. Medicine (Baltimore). 2015; 94: e966. doi: 10.1097/MD.0000000000000966

24. Buccheri G, Ferrigno D, Tamburini M. Karnofsky and ECOG performance status scoring in lung cancer: a prospective, longitudinal study of 536 patients from a single institution. Eur J Cancer. 1996; 32A: 1135-41. doi: 10.1016/0959-8049(95)00664-8.
25. Pantel $\mathrm{K}$, Brakenhoff $\mathrm{RH}, \mathrm{Brandt} \mathrm{B}$. Detection, clinical relevance and specific biological properties of disseminating tumour cells. Nature Reviews Cancer. 2008; 8: 329-40. doi:

26. Rahbari NN, Aigner M, Thorlund K, Mollberg N, Motschall E, Jensen K, Diener MK, Büchler MW, Koch M, Weitz J. Meta-analysis shows that detection of circulating tumor cells indicates poor prognosis in patients with colorectal cancer. Gastroenterology. 2010; 138: 1714-26. doi: 10.1053/j.gastro.2010.01.008.

27. Koerkamp BG, Rahbari NN, Büchler MW, Koch M, Weitz J. Circulating tumor cells and prognosis of patients with resectable colorectal liver metastases or widespread metastatic colorectal cancer: a meta-analysis. Annals of surgical oncology. 2013; 20: 2156-65. doi:

28. Hsieh JCH, Wu TMH. The Selection Strategy for Circulating Tumor Cells (CTCs) Isolation and Enumeration: Technical Features, Methods, and Clinical Applications. Tumor Metastasis: InTech). 2016. 